\title{
Optimal pebbling number of graphs with given minimum degree
}

\author{
A. Czygrinow*, G. Hurlbert $\dagger$ G. Y. Katona ${ }^{\ddagger}$ L. F. Papp ${ }^{\S}$ \\ February 22, 2017
}

\begin{abstract}
Consider a distribution of pebbles on a connected graph $G$. A pebbling move removes two pebbles from a vertex and places one to an adjacent vertex. A vertex is reachable under a pebbling distribution if it has a pebble after the application of a sequence of pebbling moves. The optimal pebbling number $\pi_{\text {opt }}(G)$ is the smallest number of pebbles which we can distribute in such a way that each vertex is reachable. It was known that the optimal pebbling number of any connected graph is at most $\frac{4 n}{\delta+1}$, where $\delta$ is the minimum degree of the graph. We strengthen this bound by showing that equality cannot be attained and that the bound is sharp. If $\operatorname{diam}(G) \geq 3$ then we further improve the bound to $\pi_{\mathrm{opt}}(G) \leq \frac{3.75 n}{\delta+1}$. On the other hand, we show that a family of graphs with optimal pebbling number $\frac{8 n}{3(\delta+1)}$ exists.
\end{abstract}

\section{Introduction}

Graph pebbling is a game on graphs initialized by a question of Saks and Lagarias, which was answered by Chung in 1989 [ 3 . Its roots are originated in number theory. Each graph in this paper is simple. We denote the vertex set and the edge set of graph $G$ with $V(G)$ and $E(G)$, respectively. We use $n$ and $\delta$ for the order and the minimum degree of $G$, respectively.

A pebbling distribution $D$ on graph $G$ is a function mapping the vertex set to nonnegative integers. We can imagine that each vertex $v$ has $D(v)$ pebbles. A pebbling move removes two pebbles from a vertex and places one to an adjacent one. We do not want to violate the definition of pebbling distribution, therefore a pebbling move is allowed if and only if the vertex loosing pebbles has at least two pebbles.

A vertex $v$ is reachable under a distribution $D$, if there is a sequence of pebbling moves, such that each move is allowed under the distribution obtained by the application of the previous moves and after the last move $v$ has at least one pebble. We say that a subgraph $H$ is solvable under distribution $D$ if each vertex of $H$ is reachable under $D$. When the whole graph is solvable under a pebbling distribution, then we say that the distribution is solvable. A pebbling distribution $D$ on a graph $G$ will be called optimal if it is solvable and $\sum_{v \in V(G)} D(v)$ is the smallest possible. The size of an optimal pebbling distribution is called the optimal pebbling number and denoted by $\pi_{o p t}(G)$.

The optimal pebbling number of several graph families are known. For example exact values were given for paths and cycles [12, 7, 11], ladders [4], caterpillars [5] and m-ary trees [6]. The values for graphs with diameter smaller than four are also characterized by some easily checkable domination conditions citesmalldiam. However, determining the optimal pebbling number for a given graph is NP-hard 9 .

In 4 the optimal pebbling number of graphs with given minimal degree is studied. This paper contains many great results about the topic. The authors proved that $\pi_{\mathrm{opt}}(G) \leq \frac{4 n}{(\delta+1)}$ and they also

\footnotetext{
*Department of Mathematics and Statistics, Arizona State University, andrzej@math.la.asu.edu.

$\dagger ? ?$ ?, Virginia Commonwealth University, ghurlbert@vcu.edu.

${ }^{\ddagger}$ Department of Computer Science and Information Theory, Budapest University of Technology and Economics, MTAELTE Numerical Analysis and Large Networks Research Group, Hungary, kiskat@cs.bme.hu.

${ }_{\S}$ Department of Computer Science and Information Theory, Budapest University of Technology and Economics, lazsa@cs.bme.hu.
} 
found a version utilizing the girth of the graph. A construction for infinite number of graphs with optimal pebbling number $\left(2.4-\frac{24}{5 \delta+15}-o\left(\frac{1}{n}\right)\right) \frac{n}{\delta+1}$ is also given in that article.

In the present paper we continue the study of graphs with fixed minimum degree. In Section 2 we present a family of graphs with arbitrary large diameter whose optimal pebbling number is $\frac{8 n}{3(\delta+1)}$. This suggests, that a sharp version of the upper bound may have the form of $\frac{c n}{\delta+1}$.

In the next part we prove a stronger upper bound when the diameter is at least three. It is shown that $\pi_{\text {opt }}(G) \leq \frac{15 n}{4(\delta+1)}$ holds in this case. Unfortunately, we do not know that if it sharp or not. Furthermore, the problem behaves differently when the diameter is two.

In Section 4.1 we show using the theory of Erdös-Rényi random graphs that for every $\epsilon>0$ there is a graph $G$ with diameter two such that $\pi_{\text {opt }}(G)>\frac{(4-\epsilon) n}{\delta+1}$. We also show that for all graphs $\pi_{\text {opt }}(G) \neq \frac{4 n}{(\delta+1)}$. These mean that this slightly stronger version of the result of Bundle et al. is sharp.

\section{A family of graphs, whose optimal pebbling number is $\frac{8 n}{3(\delta+1)}$}

We say that a vertex $v$ is dominated by a set of vertices $S$, if $v$ is contained in $S$ or there is a vertex in $S$ which is adjacent to $v$. A vertex set $S$ dominates $G$ if each vertex of $G$ is dominated by $S$. $G \square H$ denotes the Cartesian product of graphs $G$ and $H$, so $V(G \square H)=V(G) \times V(H)$ and $\left\{(g, h),\left(g^{\prime}, h^{\prime}\right)\right\} \in E(G \square H)$ if either $g=g^{\prime},\left\{h, h^{\prime}\right\} \in E(H)$ or $\left\{g, g^{\prime}\right\} \in E(G), h=h^{\prime}$.

Let $K$ be the complete graph with $V(K)=\left\{x_{1}, \ldots, x_{n}\right\}$ and let $H=\overline{K \square K}$ be the complement of $K \square K$. Two vertices are adjacent in $H$ iff both of their coordinates are different. Note that the optimal pebbling number of $H$ is four as the diameter is two and no two adjacent vertices dominate $V(H)$. Indeed if $\left(x_{i}, x_{j}\right)$ and $\left(x_{k}, x_{l}\right)$ are two adjacent vertices, then at least one of $\left(x_{i}, x_{l}\right),\left(x_{k}, x_{j}\right)$ is not dominated by them. Let $u:=\left(x_{1}, x_{1}\right), v:=\left(x_{1}, x_{n}\right)$, and $w:=\left(x_{2}, x_{2}\right)$. Then $u w v$ is an induced path in $H$. Placing two pebbles at both $u$ and $w$ creates a solvable distrubion of $H$.Let $B_{1}, \ldots, B_{3 k}$ be pairwise disjoint sets and let $H_{i}$ be a graph on $B_{i}$ which is isomorphic $H$. In addition let $u_{i}, v_{i}, w_{i}$ be vertices in $B_{i}$ which correspond to $u, v, w$ of $H$. Let $G_{3 k}$ be the graph on $\bigcup_{i=1}^{3 k} B_{i}$ and $E\left(G_{3 k}\right):=\bigcup_{i=1}^{3 k} E\left(H_{i}\right) \cup \bigcup_{i=1}^{3 k-1}\left\{v_{i} u_{i+1}\right\}$. For a pebbling function $D$ on $G_{3 k}$ and vertex $x \in V\left(G_{3 k}\right)$ we denote by $D_{L}(x)$ the number of pebbles which can be placed on $x$ using pebbles originally placed on $\bigcup_{j<i} B_{j}$. Similarly let $D_{R}(x)$ be the number of pebbles which can be placed on $x$ using pebbles originaly placed on $\bigcup_{j>i} B_{j}$. We will set $B_{i}^{\prime}:=B_{i} \backslash\left\{u_{i}, v_{i}\right\}$ and for $S \subseteq V\left(G_{3 k}\right)$ we will denote by $D(S):=\sum_{s \in S} D(s)$.

Lemma 2.1 (a) The optimal pebbling number of $G_{3}$ is 8 .

(b) If $D$ is a distribution with eight pebbles and such that $D\left(v_{3}\right)>0$, then $D$ is not solvable.

(c) If $D$ is a distribution with nine pebbles such that $D\left(v_{3}\right)>2$, then $D$ is not solvable.

Proof: First note that to solve for $B_{1}, B_{2}$ one must accumulate at least six on $B_{1} \cup B_{2}$. To prove part (a) we argue that a distribution with 7 pebbles is not solvable. Let $l:=D\left(B_{1}\right)$. If $l \geq 3$, then we can accumulate on $B_{2} \cup B_{3}$ at most $l / 2+(7-l)<6$ pebbles. If $l=0$, then 4 pebbles must be obtained from 7 on $B_{2} \cup B_{3}$ which is not possible and if $l=1$, then to be able to solve for $B_{1}$ the remaining 6 pebbles must placed on $u_{2}$ which is not solvable for $B_{3}$. Finally if $D\left(B_{1}\right)=2=D\left(B_{3}\right)$, then only 3 pebbles are on $B_{2}$ and it is not possible to move additional two to $B_{1}$. We will now prove part (b). If $D\left(v_{3}\right) \geq 1$, then either $D\left(B_{3}\right) \leq 4$ and only one pebble can be moved to $B_{1} \cup B_{2}$ from $B_{3}$ or $D\left(B_{3}\right) \geq 5$ and less than $D\left(B_{3}\right) / 2+\left(8-D\left(B_{3}\right)\right)<6$ can be accumulated on $B_{1} \cup B_{2}$. Now we prove part (c). We may assume $D\left(v_{3}\right)$ is even as otherwise we delete one pebble from $v_{3}$ and the new distribution is solvable as well. If $D\left(v_{3}\right)=: l \geq 4$, then only at most $(9-l)+l / 8=9-7 l / 8<6$ pebbles can be obtained on $B_{1} \cup B_{2}$.

Proposition 2.2 The optimal pebbling number of $G_{3 k}$ is $8 k$. 
To prove the proposition we state and prove claims stepping on each other. At each step we prove the existence of an optimal distribution having more restricted properties, then the previous one.

Claim 2.3 There exists an optimal pebbling distribution $D$ on $G_{3 k}$ such that $D(v) \leq 5$ for every vertex $v$.

Proof: Suppose that there exists a vertex $v \in B_{i}$ with $D(v) \geq 6$. Consider the distribution $D^{\prime}$ with $D^{\prime}(v):=D(v)-2$ and $D^{\prime}\left(v_{L(i)}\right):=D\left(v_{L(i)}\right)+1$ and $D^{\prime}\left(u_{R(i)}\right):=D\left(u_{R(i)}\right)+1$. Since at most one pebble out of two which were placed on $v$ can be moved to $v_{L(i)}, u_{R(i)}, D^{\prime}$ is solvable and if at least one of $L(i), R(i)$ does not exist, then $D$ is not optimal. Now apply repeatedly the above argument to obtain the property from the claim.

Claim 2.4 There exists an optimal pebbling distribution $D$ on $G_{3 k}$ such that Claim 2.3 holds and such that for every $i=1, \ldots, 3 k, D\left(B_{i}^{\prime}\right) \leq 1$ and $D\left(B_{i}^{\prime} \backslash\left\{w_{i}\right\}\right)=0$.

Proof: Fix $i \in[3 k]$ and let $l:=D\left(B_{i}^{\prime}\right)$. If $l \geq 4$, then consider $D^{\prime}$ obtained from $D$ by removing 4 pebbles and placing additional two on each of the $u_{i}$ and $v_{i}$. Clearly out of these four at most two can be placed on each and the new distribution is solvable. If $l=3$, then for $B_{i}$ to be solvable either $D\left(u_{i}\right)+D_{L}\left(u_{i}\right) \geq 1$ or $D\left(v_{i}\right)+D_{R}\left(v_{i}\right) \geq 1$. If both $D\left(u_{i}\right)+D_{L}\left(u_{i}\right) \geq 1$ and $D\left(v_{i}\right)+D_{R}\left(v_{i}\right) \geq 1$, then we remove three pebbles, place one on $w_{i}$ and additional one on each of the $u_{i}$ and $v_{i}$. Finally if $D\left(u_{i}\right)+D_{L}\left(u_{i}\right)=0$, then $D\left(v_{i}\right)+D_{R}\left(v_{i}\right) \geq 1$ and out of the three pebbles on $B_{i}^{\prime}$ at most one can be moved to $v_{i}$. We remove three pebbles from $B_{i}^{\prime}$, place two on $u_{i}$ and an additional one on $v_{i}$. Finally suppose that $l=2$. If $D\left(u_{i}\right)+D_{L}\left(u_{i}\right) \geq 1$ and $D\left(v_{i}\right)+D_{R}\left(v_{i}\right) \geq 1$, then we can place one additional pebble on each of $u_{i}, v_{i}$. If $D\left(u_{i}\right)+D_{L}\left(u_{i}\right)=0$, then $D\left(v_{i}\right)+D_{R}\left(v_{i}\right) \geq 2$ and if $D\left(v_{i}\right)+D_{R}\left(v_{i}\right) \geq 3$, then we can place one pebble on each of $u_{i}$, $v_{i}$. If on the other hand $D\left(v_{i}\right)+D_{R}\left(v_{i}\right)=2$, then $D_{R}\left(v_{i-1}\right)=0$ and we can place two additional pebbles on $v_{i}$.

Claim 2.5 There exists an optimal pebbling distribution $D$ on $G_{3 k}$ such that Claims 2.32 .4 hold and such that $D(v) \leq 4$ for every vertex $v$.

Proof: Assume that $D\left(u_{i}\right)=5$. If $D\left(v_{i}\right)+D_{R}\left(v_{i}\right) \geq 1$, then we can take two pebbles from $u_{i}$, place one of them on $v_{i}$ and another one on $v_{L(i)}$. The new distribution is solvable. Assume therefore that $D\left(v_{i}\right)+D_{R}\left(v_{i}\right)=0$. In this case $D_{R}\left(v_{i-1}\right)=2$ and we can remove one pebble from $u_{i}$ and place it on $v_{i}$.

Block $B_{i}$ is supersaturated if the sum of $D\left(u_{i}\right)$ and $D\left(v_{i}\right)$ is at least 5 or one of values is 4 . Note that a supersaturated block is solvable using its own pebbles.

Claim 2.6 There exists an optimal pebbling distribution $D$ on $G_{3 k}$ such that Claims 2.32 .2 .5 hold and such that $D\left(u_{i}\right)+D\left(v_{i}\right) \leq 5$ and moreover if $D(v)=4$ for some $v \in B_{i}$, then $D(B \backslash\{v\})=0$.

Proof: Assume that $D\left(u_{i}\right) \geq 3$ and $D\left(v_{i}\right) \geq 3$, then we can take a pebble from each and move one to the left and one to the right to first non-supersaturated blocks. For the second part, suppose that $D\left(u_{i}\right)=4$. If $D\left(v_{i}\right)+D_{R}\left(v_{i}\right) \geq 1$, then take two pebbles from $u_{i}$ place an additional one on $v_{i}$ and move one to the first non-supersaturated blocks to the left of $B_{i}$. If $D\left(v_{i}\right)+D_{R}\left(v_{i}\right)=0$ but $D\left(w_{i}\right)=1$, then take the pebble off $w_{i}$ and two off $u_{i}$. Move one to the left and place two on $v_{i}$.

Lemma 2.7 If $D$ is such that Claims 2.3 2.6 hold, then for every $u_{i}, v_{i}, D_{L}\left(u_{i}\right) \leq 2$ and $D_{R}\left(v_{i}\right) \leq 2$. 
Proof: We argue by induction. To show $D_{L}\left(u_{i+1}\right) \leq 2$, we can assume that either $D\left(u_{i}\right)=2$ and $D\left(v_{i}\right)=3$ or $D\left(u_{i}\right)=0$ and $D\left(v_{i}\right)=4$. Since $D_{L}(u) \leq 2$ be the inductive assumption at most two pebbles can be moved from $v_{i}$ to $u_{i+1}$.

Block $B_{i}$ is called special if $D\left(w_{i}\right)=D\left(u_{i}\right)=D\left(v_{i}\right)=D_{L}\left(u_{i}\right)=D_{R}\left(v_{i}\right)=1$.

Claim 2.8 There exists an optimal pebbling distribution $D$ on $G_{3 k}$ such that Claims $2.3 \sqrt{2.6}$ hold and such that for every $D\left(B_{i}^{\prime}\right)=0$.

Proof: Assume that $D\left(w_{i}\right)=1$. We may assume that $D\left(u_{i}\right) \leq 2$ and $D\left(v_{i}\right) \leq 3$. First suppose that $D\left(v_{i}\right)+D_{R}\left(v_{i}\right) \geq 3$. If $D\left(u_{i}\right)=0$, then as $D\left(v_{i}\right)+D_{R}\left(v_{i}\right) \leq 5$ we have $D_{R}\left(v_{i-1}\right)=0$. We can then remove the pebble from $w_{i}$ and put it on $v_{i}$. If $D\left(u_{i}\right)=1$, then we remove the pebble from $w_{i}$ and one pebble from $v_{i}$, put an additional pebble on $u_{i}$ and move one pebble to the first non-supersaturated block $B_{j}$ with $j>i$. Finally if $D\left(u_{i}\right)=2$, then we remove the pebble from $w_{i}$ and one from $v_{i}$ move one pebble to the first non-supersaturated block on the right and on the left. Thus $D\left(v_{i}\right)+D_{R}\left(v_{i}\right) \leq 2$ and similarly $D\left(u_{i}\right)+D_{L}\left(u_{i}\right) \leq 2$. If $D\left(u_{i}\right)+D_{L}\left(u_{i}\right) \neq 2$, then we can move the pebble from $w_{i}$ to $u_{i}$ to obtain a solvable distribution. Thus both $D\left(u_{i}\right)+D_{L}\left(u_{i}\right)$ and $D\left(v_{i}\right)+D_{R}\left(v_{i}\right)$ equal to 2 . In which case $D_{R}\left(v_{i-1}\right) \leq 1$ and $D_{L}\left(u_{i+1}\right) \leq 1$. If $D\left(u_{i}\right)=2$, then $D_{R}\left(v_{i-1}\right)=1$ and we can move the pebble from $w_{i}$ to $v_{i}$. If $D\left(u_{i}\right)=0$, then $D_{R}\left(v_{i-1}\right)=0$ and we can move the pebble from $w_{i}$ to $v_{i}$. Thus we may assume that $D\left(u_{i}\right)=1$ and similarly $D\left(v_{i}\right)=1$ and so $B_{i}$ is special. If $B_{i}$ and $B_{i-1}$ are special, then we delete all pebbles from $B_{i-1} \cup B_{i}$ place three pebbles on each of the $u_{i-1}$ and $v_{i}$. Therefore we may assume that neither $B_{i-1}$ nor $B_{i+1}$ are special. Since $D_{L}\left(u_{i}\right)=D_{R}\left(v_{i}\right)=1$ we have $1 \leq D\left(v_{i-1}\right), D\left(u_{i+1}\right) \leq 3$. Suppose $D\left(v_{i-1}\right)=3$. If $D\left(u_{i-1}\right)=0$, then we can remove the pebble from $u_{i}$ place it on $v_{i-1}$ and remove the pebble from $w_{i}$ and place it on $v_{i}$. If on the other hand $D\left(u_{i-1}\right) \geq 1$, then we can remove one pebble from $v_{i-1}$ and place it on $u_{i-1}$. Consequently we may assume that that $D\left(v_{i-1}\right), D\left(u_{i+1}\right) \leq 2$. $B_{i-1}$ is not special, hence if $D\left(v_{i-1}\right)=1$, then $D\left(u_{i-1}\right)+D_{L}\left(u_{i-1}\right) \geq 4$ for $D_{L}\left(u_{i}\right)=1$ to hold. In which case we can move the pebble from $v_{i-1}$ to $u_{i}$ and the pebble from $w_{i}$ to $v_{i}$. Finally if $D\left(v_{i-1}\right)=D\left(u_{i+1}\right)=2$, then $D_{L}\left(u_{i-1}\right)+D\left(u_{i-1}\right)+D\left(w_{i-1}\right), D_{R}\left(v_{i+1}\right)+D\left(v_{i+1}\right)+D_{R}\left(w_{i+1}\right) \geq 2$ and we can remove the pebble from $w_{i}$.

A block $B_{i}$ is called good if either $D\left(u_{i}\right)=4$ or $D\left(v_{i}\right)=4$ or $D\left(u_{i}\right), D\left(v_{i}\right) \leq 2$. A block is called saturated if either $D\left(u_{i}\right)=4$ or $D\left(v_{i}\right)=4$ or $D\left(u_{i}\right)=2, D\left(v_{i}\right)=2$. Note that if the block is saturated, then $B_{i}$ is solvable using pebbles from $B_{i}$.

Claim 2.9 There exists an optimal pebbling distribution $D$ on $G_{3 k}$ such that Claims $2.3 \sqrt{2.8}$ hold and such that each block is good.

Proof: Assume that $D\left(u_{i}\right)=3$ and $D\left(v_{i}\right) \leq 2$. Then $D_{L}\left(u_{i+1}\right) \leq 1$. If $D\left(v_{i}\right)=2$, then we can remove one pebble from $u_{i}$ and move it on the first non-saturated block to the left of $B_{i}$. If $D\left(v_{i}\right)+D_{R}\left(v_{i}\right) \geq 1$, then as $D_{R}\left(v_{i-1}\right)=1$, we can move one pebble from $u_{i}$ to $v_{i}$. If $D\left(v_{i}\right)+D_{R}\left(v_{i}\right)=0$, then $D_{L}\left(u_{i}\right) \geq 1$. If $D_{L}\left(u_{i}\right)=1$, then $1 \leq D\left(v_{i-1}\right) \leq 3$ and so we can take one pebble from $v_{i-1}$ and add it to $u_{i}$. If $D_{L}\left(u_{i}\right)=2$, then we can remove one pebble from $u_{i}$ and move it to the first non-saturated block to the left.

Lemma 2.10 If a pebbling distribution $D$ is such that Claims 2.3 2.9 hold, then $D_{L}\left(u_{i}\right)>1$ implies that $D\left(v_{i-1}\right)=4$.

Proof. This follows by induction.

Claim 2.11 There exists an optimal pebbling distribution $D$ on $G_{3 k}$ such that Claims $2.3 \sqrt{2.9}$ hold and such that $D\left(B_{1} \cup B_{2} \cup B_{3}\right)=8$ and $D_{L}\left(u_{4}\right)=0$. 
Proof: First suppose that $D\left(B_{1} \cup B_{2} \cup B_{3}\right) \geq 10$. Then we can take all but 8 pebbles and move them to the first non-saturated block to the right and rearrange the eight pebbles to obtain both properties. If $D\left(B_{1} \cup B_{2} \cup B_{3}\right) \leq 6$, then as $D_{R}\left(u_{i}\right) \leq 2$ the distribution is not solvable by Lemma 2.1 (b). If $D\left(B_{1} \cup B_{2} \cup B_{3}\right)=7$, then from Lemma 2.1 (b), $D\left(u_{4}\right)=4$ and so $D\left(v_{4}\right)=0$. By Lemma 2.1 (c) $D\left(v_{3}\right)=0$ and so $D_{L}\left(u_{4}\right)=0$. First assume that $D\left(B_{5} \cup B_{6}\right) \geq 6$. If $D_{L}\left(u_{7}\right) \leq 1$, then we can take two arbitrary pebbles from $B_{5} \cup B_{6}$ and move one of them to the first non-saturated block $B_{j}$ with $j \geq 7$ and one to the first three blocks to obtain eight pebbles there and rearrange them. Furthermore we move the 4 pebbles of $u_{4}$ to $v_{4}$ and place 4 pebbles of $B_{5} \cup B_{6}$ at $u_{6}$ to make $B_{5} \cup B_{6}$ solvable. If $D_{L}\left(u_{7}\right)=2$, then $D\left(v_{6}\right)=4$ and so $D\left(u_{6}\right)=0$. Thus for $B_{5}$ to be solvable we must have $D\left(B_{5}\right) \geq 4$. Take one pebble from $B_{5}$ and move it to the first three block rearranging $D$ so that the first three blocks are solvable. Put $D\left(B_{5}\right)-1$ pebbles on $v_{5}$ and move 4 pebbles from $u_{4}$ to $v_{4}$. The new distribution is solvable for $B_{5}$. If $D\left(B_{5} \cup B_{6}\right)=5$, then either $D\left(B_{5}\right)=3$ and $D\left(u_{6}\right)=2$ or $D\left(B_{5}\right)=4$ and in either case $D_{L}\left(u_{7}\right)=0$. Thus we can rearrange all 16 pebbles on $B_{1} \cup \cdots \cup B_{6}$. If finally $D\left(B_{5} \cup B_{6}\right)=4$, then $D\left(B_{5}\right)=4$ and consequently for $B_{6}$ to be solvable, $D\left(u_{7}\right)=4$. Then we can repeat the analysis for the next three blocks and either move one to the first three and rearrange or continue. Since $D$ is solvable we cannot continue indefinitely as the last three blocks cannot be solved. If $D\left(B_{1} \cup B_{2} \cup B_{3}\right)=9$ and $D_{L}\left(u_{4}\right)=1$, then we can move one out of 9 and rearrange. If on the other hand $D_{L}\left(u_{4}\right)=2$, then $D\left(v_{3}\right)=4$ and so $D\left(u_{3}\right)=0$. Thus $D_{R}\left(v_{2}\right)=0$ and only 5 pebble are placed in the first two blocks so the distribution is not solvable. Therefore $D\left(B_{1} \cup B_{2} \cup B_{3}\right)=8$. Now assume that $D_{L}\left(u_{4}\right) \geq 1$. If $D_{L}\left(u_{4}\right) \geq 2$, then again $D\left(v_{3}\right)=4$ and the distribution is not solvable. If $D_{L}\left(u_{4}\right)=1$, then $D\left(v_{3}\right) \geq 1$ and so ,by Lemma 2.1 (b), for $D$ to be solvable for $B_{1} \cup B_{2} \cup B_{3}$ we must have $D_{R}\left(v_{3}\right) \geq 1$. If $D_{R}\left(v_{3}\right)=2$, then $D\left(u_{4}\right)=4$ and we can rearrange by moving 4 pebbles from $u_{4}$ to $v_{4}$ and rearranging 8 on $B_{1} \cup B_{2} \cup B_{3}$ so that $D_{L}\left(u_{4}\right)=0$. Thus $D_{R}\left(v_{3}\right)=1$ and $D\left(v_{3}\right) \geq 1$. If $D\left(v_{3}\right) \geq 2$, then the distribution is not solvable for $B_{1} \cup B_{2} \cup B_{3}$ by Lemma 2.1 (c). If $D\left(v_{3}\right)=1$, then $D\left(u_{3}\right)+D_{L}\left(u_{3}\right) \geq 4$ for $D_{L}\left(u_{4}\right) \geq 1$. Thus $D\left(u_{3}\right)=2$ which gives $D_{R}\left(v_{2}\right)=1$ and $D\left(v_{2}\right)=4$. Then however $D\left(B_{1}\right) \leq 1$ and we cannot solve for $B_{1}$. Consequently $D\left(B_{1} \cup B_{2} \cup B_{3}\right)=8$ and $D_{L}\left(u_{4}\right)=0$.

Proof of 2.2. We prove by induction on $k$ that the optimal pebbling number is at least $8 k$. The base case was established in Lemma 2.1. Let $D$ be a distribution on $G_{3 k}$ satisfying $D\left(B_{1} \cup B_{2} \cup B_{3}\right)=8$ and $D_{L}\left(u_{4}\right)=0 . D$ restricted to $B_{4} \cup \cdots \cup B_{3 k}$ must be solvable for $B_{4} \cup \cdots \cup B_{3 k}$ and so by induction hypothesis it must have at least $8(k-1)$ pebbles.

\section{Improved upper bound when diameter is at least three}

In this section we give a construction of a pebbling distribution having at most $\frac{15 n}{4(\delta+1)}$ pebbles for any graph whose diameter is at least three.

The distance between vertices $u$ and $w$ is the number of edges contained in the shortest path between them. We denote this quantity with $d(u, v)$. The distance- $k$ open neighbourhood of a vertex $v$, denoted by $N^{k}(v)$, contains all vertices whose distance from $v$ is exactly $k$. On the other hand, the distance- $k$ closed neighbourhood of $v$ contains all vertices whose distances from $v$ is at most $k$. We denote this set with $N^{k}[v]$. When $k=1$ we omit the distance- 1 part from the name and the upper index 1 from the notation.

We are going to talk about several graphs on the same labeled vertex set. To make it clear which graph we are considering in a formula we write the name of the graph as a lower index, i.e. $d_{G}(u, v)$ is the distance between vertices $u$ and $v$ in graph $G$.

We define distances between subgraphs in the natural way: If $H$ and $K$ are subgraphs of $G$, then $d_{G}(H, K)=\min _{u \in V(H), v \in V(K)}\left(d_{G}(u, v)\right)$.

We can think about a vertex as a subgraph, therefore let distance- $k$ open neighbourhood of a subgraph $H$ be the set of vertices whose distance from $H$ is exactly $k$. We define the closed neighbourhood similarly. Note that $N^{d}(H)=N^{d}[H] \backslash N^{d-1}[H]$. 
The following property will be useful in our investigations: A vertex $v \in V(G)$ is strongly reachable under $D$ if each vertex from the closed neighborhood of $v$ is reachable under $D$. This property together with traditional reachabilty partition the vertex set to three sets $\mathcal{T}(D), \mathcal{H}(D)$ and $\mathcal{U}(D)$, where $\mathcal{T}(D)$ includes the strongly reachable vertices, the vertices of $\mathcal{H}(D)$ are reachable but not stronly reachable and $\mathcal{U}(D)$ contains the rest of the vertices.

Theorem 3.1 Let $G$ be a connected graph, such that its diameter is bigger than two and $\delta$ is its minimum degree. We have

$$
\pi_{\text {opt }}(G) \leq \frac{15 n}{4(\delta+1)}
$$

Let $D$ and $D^{\prime}$ be pebble distributions. $D^{\prime}$ is an expansion of $D^{\prime}\left(D \leq D^{\prime}\right)$ if $\forall v \in V(G) D(v) \leq D^{\prime}(v)$. If $D \neq D^{\prime}$, then we write that $D<D^{\prime}$. If $D^{\prime}$ is an expansion of $D$, then let $\Delta_{D, D^{\prime}}$ be the pebbling distribution defined as: $\Delta_{D, D^{\prime}}(v)=D^{\prime}(v)-D(v) \forall v \in V(G)$.

If we would like to create a solvable distribution, then we can do it incrementally. We start with the trivial distribution with no pebbles and add more and more pebbles to it. So we have a sequence of distributions $0<D_{1}<D_{2}<\cdots<D_{k-1}<D_{k}$ where $D_{k}$ is solvable. The number of reachable vertices is growing during this process. We can ask which vertices are reachable, strongly reachable, or not reachable after the $i$ th step. Let $\mathcal{T}\left(D_{i}\right), \mathcal{H}\left(D_{i}\right), \mathcal{U}\left(D_{i}\right)$ denote these sets respectively. Note that $\mathcal{T}\left(D_{i}\right) \subseteq \mathcal{T}\left(D_{i+1}\right)$, while $\mathcal{U}\left(D_{i}\right) \supseteq \mathcal{U}\left(D_{i+1}\right)$. Furthermore we know that $\mathcal{T}\left(D_{k}\right)=V(S)$ and $\mathcal{H}\left(D_{k}\right)=\mathcal{U}\left(D_{k}\right)=\emptyset$.

If for each $i$ the difference $\left|\Delta_{D_{i}, D_{i+1}}\right|$ is relatively small and $\left|\mathcal{T}\left(D_{i+1}\right) \backslash \mathcal{T}\left(D_{i}\right)\right|$ is relatively big, then it yields that $\left|D_{k}\right|$ is not so big.

To make this intuitive idea precise we define the strengthening ratio.

Definition 3.2 Suppose that we have distributions $D$ and $D^{\prime}$ on graph $G$, such that $D<D^{\prime}$. Denote the difference of the size of these distribution by $\Delta p_{D, D^{\prime}}=\left|D^{\prime}\right|-|D|=\left|\Delta_{D, D^{\prime}}\right|$. We use $\Delta \mathcal{T}_{D, D^{\prime}}$ for set $\mathcal{T}\left(D^{\prime}\right) \backslash \mathcal{T}(D)$ and $\Delta t_{D, D^{\prime}}$ denotes the cardinality of this set.

We say that the strengthening ratio of the expansion $D<D^{\prime}$ is:

$$
\mathcal{E}\left(D, D^{\prime}\right)=\frac{\Delta t_{D, D^{\prime}}}{\Delta p_{D, D^{\prime}}}
$$

The strengthening ratio of distribution $D \neq 0$ is $\mathcal{E}(0, D)$, and the strengthening ratio of $D=0$ is $\infty$.

Fact 3.3 If $D$ is solvable, then $|D|=\frac{n}{\mathcal{E}(0, D)}$.

This fact shows that if we want to give a solvable distribution whose size is close to the optimum, then its strengthening ratio is also close to the optimum. Furthermore, a smaller solvable distribution has bigger strengthening ratio. The next lemma shows that if we break $D_{k}$ to a sequence of expansions $0<D_{1}<D_{2}<\cdots<D_{k-1}<D_{k}$, then the strengthening ratio of each expansion is a lower bound for $\mathcal{E}\left(0, D_{k}\right)$. Therefore we are looking for an expansion chain where the minimum strengthening ratio among all expansion steps is relatively big.

Lemma 3.4 Let $D_{1}, D_{2}$ and $D_{3}$ are distributions on $G$. If $D_{1}<D_{2}$ and $D_{2}<D_{3}$, then

$$
\mathcal{E}\left(D_{1}, D_{3}\right) \geq \min \left(\mathcal{E}\left(D_{1}, D_{2}\right), \mathcal{E}\left(D_{2}, D_{3}\right)\right) .
$$

Proof:

Let $a, b, c, d$ be nonnegative real numbers, then the following inequality can be easily proven by elementary tools:

$$
\frac{a+b}{c+d} \geq \min \left(\frac{a}{c}, \frac{b}{d}\right)
$$


Useing this and the definition of strengthening ratio, we obtain

$$
\begin{aligned}
\mathcal{E}\left(D_{1}, D_{3}\right) & =\frac{\Delta t_{D_{1}, D_{3}}}{\Delta p_{D_{1}, D_{3}}}=\frac{\Delta t_{D_{1}, D_{2}}+\Delta t_{D_{2}, D_{3}}}{\Delta p_{D_{1}, D_{2}}+\Delta p_{D_{2}, D_{3}}} \geq \\
& \geq \min \left(\frac{\Delta t_{D_{1}, D_{2}}}{\Delta p_{D_{1}, D_{2}}}, \frac{\Delta t_{D_{2}, D_{3}}}{\Delta p_{D_{2}, D_{3}}}\right)=\min \left(\mathcal{E}\left(D_{1}, D_{2}\right), \mathcal{E}\left(D_{2}, D_{3}\right)\right) .
\end{aligned}
$$

In the next lemma we state that we can construct a distribution $D$ with some special properties. This lemma formalizes the following idea: If there are pairs of adjacent vertices, such that the closed neighborhood of each pair is large, then we can make all vertices of these pairs reachable with few pebbles, while lots of other vertices become reachable. The connection between few and lots of is established by strengthening ratio.

Lemma 3.5 Let $G$ be an arbitrary simple connected graph. There is a pebbling distribution $D$ on $G$ which satisfies the following conditions:

1. The strengthening ratio of $D$ is at least $\frac{4}{15}(\delta+1)$.

2. If $(u, v)$ is an edge of $G$ and $|N[u] \cup N[v]| \geq \frac{29}{15}(\delta+1)$, then both of $u$ and $v$ are reachable under D.

Proof: Our proof is a construction for such a $D$ :

We say that and edge $(u, v)$ has * property iff $|N[u] \cup N[v]| \geq \frac{29}{15}(\delta+1)$.

First of all, if there is no $(u, v)$ edge in $G$ with * property, then the trivial distribution 0 is good to be $D$. Otherwise, we have to make reachable each vertex of any edge which has ${ }^{*}$ property. To make this we search for these edges, and if we find such an edge such that at least one of its vertices is not reachable, then we add some pebbles on $D$ to make it reachable.

We will define sets $H, A, B \subset V(G), P, R \subset V(G) \square V(G)$ and let $L_{p}$ be a set containing vertices of $G$ for each $p \in P$.

These sets, except $H$, will contain the edges with * property or their vertices. They will have the following semantics at the end of the construction:

- Each element of $H$ will be reachable under $D$, but not necessarily all of the reachable vertices contained in it.

- Each vertex of $B$ has a neighbor who has at least two 4-reachable distance-2 neighbors, or has an 8-reachable distance-3 neighbor.

- The elements of $P$ are edges whose vertices will be 4-reachable.

- The elements of $R$ are edges whose vertices will be 8-reachable.

- $L_{p}$ contains vertices from $A$ whose distance from $p$ is exactly 3 .

Then do the following:

1. Choose an edge $(u, v)$ which has * property and $u, v \notin H$. If we can not choose such an edge, then move to step 3 .

2. Add the elements of $N^{2}[u] \cup N^{2}[v]$ to $H$. Add $(u, v)$ to $P$. Move to step 1 .

3. Search for an edge $(u, v)$ which has $*$ property and $v \notin H$. If we can not find one, then move to step 6 . 
4. Add the elements of $N^{2}[v]$ to $H$.

5. Count the number of pairs $p$ in $P$ whose distance from $u$ is 2 . If we get more than one, then add $v$ to $B$ and move to step 3. Otherwise, add $v$ to $A$ and add $v$ to the set $L_{p}$ where $p$ is the only pair whose distance from $u$ is 2 .

6. Do for each $p \in P$ : If $\left|L_{p}\right| \geq 5$, then move the elements of $L_{p}$ from $A$ to $B$ and also move $p$ from $P$ to $R$.

7. Let $D$ be the following:

$$
D(v)= \begin{cases}4 & \text { if } v \in A, \\ 3 & \text { if either } v \in B \text { or } v \text { is an element of pair } p \in P, \\ 5 & \text { if } v \text { is the first element of pair } p \in R \\ 6 & \text { if } v \text { is the second element of pair } p \in R, \\ 0 & \text { otherwise. }\end{cases}
$$

First, if we choose an edge with * property, then both vertices of it are reachable under $D$. To see this consider $H$. Each vertex of $H$ is reachable under $D$ by construction. We expanded $H$ by distance-2 closed neighborhoods of vertices which are 4-reachable in each step. Each vertex of an edge with * property is contained in $H, A$ or $B$.

Hence the second condition is satisfied. So we just need to verify the first one.

The vertices of sets $A, B$, and vertices of edges contained in $P$ and $R$ are all 4-reachable. Hence each vertex belongs to their neighborhood is strongly reachable. This implies that:

$$
\Delta t_{0, D}=|\mathcal{T}(D)| \geq\left|\left(\bigcup_{p \in P \cup R} N[p]\right) \bigcup\left(\bigcup_{v \in A \cup B} N[v]\right)\right|=\sum_{p \in P \cup R}|N[p]|+\sum_{v \in A \cup B}|N[v]|
$$

For the second equality we need that these neighborhoods are disjoint, but this is true because of the construction: The distance between a vertex of $A \cup B$ and a pair $p$ of $P \cup R$ is at least 3. The distance between $p, p^{\prime} \in P \cup R$ is also at least 3. Both of these are guaranteed by step 2. $d(u, v) \geq 3$ where $u, v \in A \cup B$ because of step 4 .

Using the ${ }^{*}$ property of edges contained in $P$ and $R$ gives:

$$
\begin{gathered}
\Delta t_{0, D} \geq \sum_{p \in P \cup R}|N[p]|+\sum_{v \in A \cup B}|N[v]| \geq(|P|+|R|) \cdot \frac{29}{15}(\delta+1)+(|A|+|B|)(\delta+1), \\
\Delta p_{0, D}=|D|=4|A|+3|B|+6|P|+11|R|, \\
\mathcal{E}(0, D)=\frac{\Delta t_{0, D}}{\Delta p_{0, D}} \geq \frac{(|P|+|R|) \cdot \frac{29}{15}(\delta+1)+(|A|+|B|)(\delta+1)}{4|A|+3|B|+6|P|+11|R|}
\end{gathered}
$$

Using $(a+b) /(c+d) \geq \min (a / c, b / d)$ :

$$
\mathcal{E}(0, D) \geq \min \left(\frac{\left(\frac{29}{15}|P|+|A|\right)(\delta+1)}{6|P|+4|A|}, \frac{\left(\frac{29}{15}|R|+|B|\right)(\delta+1)}{11|R|+3|B|}\right) .
$$

Step 6 of the construction implies that $|A| \leq 4|P|$ and $|B| \geq 5|R|$.

Let $|A|=4 x|P|$. In this case $0 \leq x \leq 1$ and we get the following function of $x$ for the first part, which gains its minimum at $x=1$ :

$$
\frac{\left(\frac{29}{15}|P|+|A|\right)(\delta+1)}{6|P|+4|A|}=\frac{\left(\frac{29}{15}+4 x\right)(\delta+1)}{6+16 x} \geq \frac{89}{330}(\delta+1) .
$$


Let $|B|=5 y|R| .|B| \geq 5|R|$ implies that $1 \leq y$. The function which we get from the second part gains its minimum at $y=1$ :

$$
\frac{\left(\frac{29}{15}|R|+|B|\right)(\delta+1)}{11|R|+3|B|}=\frac{\left(\frac{29}{15}+5 y\right)(\delta+1)}{11+15 y} \geq \frac{4}{15}(\delta+1) .
$$

This completes the proof of the Lemma.

During the proof we will show that a non solvable distribution whose strengthening ratio is above the desired bound always can be expanded to a bigger one whose strengthening ratio is still reasonable. To do this we want to decrease the number of vertices which are not strongly reachable. Usually we place some pebbles at not reachable vertices. We know that if a vertex $v$ is not reachable under $D$ and we make it 4-reachable, then all vertices of its closed neighborhood, which were not strongly reachable, become strongly reachable.

We usually consider a connected component $S$ of the graph induced by $\mathcal{U}(D)$.

There are several reasons why we do this. First of all, a chosen $S$ is a small connected part of $G$ where none of the vertices are reachable, hence it is much simpler to work with $S$ instead of the whole graph.

A vertex from $S$ has the property that none of its neighbors are strongly reachable. Thus, if we make a vertex from $S$ 4-reachable, then its whole closed neighborhood becomes strongly reachable.

Another reason for considering such an $S$ is that if we add some additional pebbles to $S$ and make sure that all of its vertices become reachable, then these vertices become strongly reachable, too.

If we make $u$ and $v$ both 4-reachable with at most 7 pebbles and their closed neighborhoods are disjoint then this is good for us. The disjointness of the neighborhoods happens when $d(v, u) \geq 3$.

We said that we want to investigate $S$, which is a connected component of $\mathcal{U}(D)$. On the one hand, it is beneficial, but on the other hand it makes some trouble when we consider distances. Let $u$ and $v$ be vertices of $S$. Their distance can be different in $G$ and $S$. For example if $G$ is the wheel graph on $n$ vertices and we place just one pebble at the center vertex, then $S$ is the outer circle and the distance between two vertices of $S$ can be $\left\lfloor\frac{n-1}{2}\right\rfloor$, while their distance in $G$ is not larger than 2 .

This difference is important because this shows that we can not decide the disjointness of closed neighborhoods by distance induced by $S$. The first idea to handle this is considering the original distance given by $G$, but then we have to consider the whole graph, which we would like to avoid. To overcome this problem we make the following compromise:

We count distances in graph $N[S]$. Clearly, this distance also can be smaller than the corresponding distance in $G$, but it happens only for values higher than 3. Hence this $N[S]$ distance determine disjointness of the neighborhoods, and it will be enough for our investigation.

The following lemmas will be used in the proof.

Fact 3.6 Let $S$ and $B$ be induced subgraphs of $G$ such that $V(B)=N_{G}[V(S)]$. If $\max _{u, v \in V(S)}\left(d_{B}(u, v)\right)=3$, then either there exist vertices $a, b, c, d \in V(S)$, such that they are neighbors in this order and $d_{B}(a, d)=$ 3 , or there exist vertices $a, d \in V(S)$ such that $d_{B}(a, d)=3$ and there is a path $P$ between a and $d$ whose length is 3 and $P$ contains a vertex from $V(B) \backslash V(S)$.

Lemma 3.7 Let $\delta$ be the minimum degree of graph $G$. Let $S$ and $B$ are connected induced subgraphs of $G$, such that $V(B)=N_{G}[V(S)]$. If $\max _{u, v \in V(S)}\left(d_{B}(u, v)\right)=3$ and exist $a, d \in V(S)$, such that $d_{B}(a, d)=d_{S}(a, d)=3$, then there is an $u, v$ edge in $S$ whose closed neighborhood has size at least $\frac{4}{3}(\delta+1)$.

Proof: Let $a, b, c, d$ be the vertices of a shortest path between $a$ and $d$ which lies in $S$. If the statement holds for edge $(a, b)$ or $(c, d)$, then we have found the edge which we are looking for. Thus assume the contrary. The Inclusion-exclusion principle gives us the following result for vertex pair $a, b$ :

$$
|N[a] \cap N[b]|=\underbrace{|N[a]|}_{\geq \delta+1}+\underbrace{|N[b]|}_{\geq \delta+1}-\underbrace{|N[a] \cup N[b]|}_{<\frac{4}{3} \delta+\frac{4}{3}}>\frac{2}{3} \delta+\frac{2}{3} .
$$


The same is true for pair $c, d$.

The distance of $a, d$ implies that $N[a] \cap N[d]=\emptyset$. Thus $(N[a] \cap N[b]) \cap(N[c] \cap N[d])=\emptyset$

$$
\begin{aligned}
|N[b] \cup N[c]| & \geq|(N[b] \cap N[a]) \cup(N[c] \cap N[d])|= \\
& =|N[b] \cap N[a]|+|N[c] \cap N[d]|-|(N[a] \cap N[b]) \cap(N[c] \cap N[d])|> \\
& >2\left(\frac{2}{3} \delta+\frac{2}{3}\right)-0=\frac{4}{3} \delta+\frac{4}{3} .
\end{aligned}
$$

So edge $b, c$ has the required property.

Lemma 3.8 Let $S$ and $B$ be connected induced subgraphs of $G$, such that $V(B)=N_{G}[V(S)]$. Assume that there are vertices $u$ and $v$ in $S$ whose distance in $B$ is 4 . Then at least one of the following conditions holds:

1. There exists $a, b \in V(S)$, such that $d_{S}(a, b)=d_{B}(a, b)=4$

2. There exists $c, d \in S$, such that $d_{B}(c, d)=3$ and some of the shortest paths between $c, d$ contain a vertex from set $V(B) \backslash V(S)$.

Proof: Consider a pair of vertices $u, v \in V(S)$ whose distance in $B$ is four. It is clear that $d_{S}(u, v) \geq 4$. Equality means that the first condition is fulfilled. Assume that the distance in $S$ between $u$ and $v$ is greater than four. Let $P$ be a shortest path between $u$ and $v$ which lies in $S$. The length of $P$ is at least five. Label the vertices of $P$ as $u=p_{0}, p_{1}, p_{2}, \ldots, p_{k}=v$. Let $i$ be the smallest value such $p_{i}$ does not have a neighbor in $N_{B}[u]$. The minimality of $i$ implies that $d_{B}\left(u, p_{i}\right)=3$. If $i>3$, then the shortest path between $u$ and $p_{i}$, which has length three, has to contain a vertex from $V(B) \backslash V(S)$. This gives us the second condition.

Otherwise $i=3$. Let $j$ be the smallest value, such that $p_{j}$ does not have a neighbor in $N_{B}^{2}[u]$. The case $j=4$ gives us $d_{B}\left(u, p_{j}\right)=4=d_{S}\left(u, p_{j}\right)$ which fulfills the first condition. The other case is $j>4$, when $d_{B}\left(p_{0}, p_{4}\right)=3$. It can happen if and only if the second condition holds.

Lemma 3.9 Let $\delta$ be the minimum degree of $G, S$ and $B$ be induced subgraphs of $G$, such that $V(B)=$ $N[V(S)]$. If $\max _{u, v \in V(S)}\left(d_{B}(u, v)\right) \geq 4$ and exist vertices a, $e \in V(S)$ such that $d_{B}(a, e)=d_{S}(a, e)=4$, then one of the following two conditions holds:

1. There exist $u, v \in S$ such that $d_{B}(u, v)=2$ and $\left|N_{B}[u] \cup N_{B}[v]\right| \geq \frac{28}{15}(\delta+1)$.

2. $|N[a] \cup N[e] \cup(N[b] \cap N[d])| \geq \frac{32}{15}(\delta+1)$, where $a, b, c, d$, e are the vertices of a path lying in $S$.

Proof: Assume that 1. does not hold. This gives us the following estimate on the size of the common neighborhood of $b$ and $d$ :

$$
\left|N_{G}[b] \cap N_{G}[d]\right|=\underbrace{\left|N_{G}[b]\right|}_{\geq \delta+1}+\underbrace{\left|N_{G}[d]\right|}_{\geq \delta+1}-\underbrace{\left|N_{G}[b] \cup N_{G}[d]\right|}_{<\frac{28}{15}(\delta+1)}>\frac{2}{15}(\delta+1)
$$

since $a$ and $d$ do not have a common neighbor. The same is true for the pairs of $b, e$, and $a, e$ which implies:

$$
|N[a] \cup N[e] \cup(N[b] \cap N[d])|=|N[a]|+|N[e]|+|N[b] \cap N[d]| \geq \frac{32}{15}(\delta+1) .
$$

So if 1. does not hold, then 2, does.

The next lemma will be useful to give a lower bound on the number of vertices becoming strongly reachable after the addition of some pebbles to $S$. 
Lemma 3.10 Let $D$ be a pebbling distribution on $G$. Let $S$ be a connected component of the subgraph which is induced by $\mathcal{U}(D)$. Consider $D^{\prime}$ such that $D \leq D^{\prime}$. If there is an $s \in V(S)$ such that $s$ is 2-reachable under $\Delta_{D, D^{\prime}}$ and each vertex of $S$ is reachable under $D^{\prime}$, then $N[s] \subseteq \mathcal{T}\left(D^{\prime}\right)$, furthermore $N[s] \subseteq \Delta \mathcal{T}_{D, D^{\prime}}$.

Proof: We show that each neighbor of $s$ is strongly reachable under $D^{\prime}$. Let $v$ be a neighbor of $s$, and $u$ be a neighbor of $v . s$ is 2-reachable under $D^{\prime}$ hence $v$ is reachable.

If $u$ is reachable under $D$ or it is a vertex of $S$, then it is reachable under $D^{\prime}$. Else $u$ is in $\mathcal{U}(D) \backslash V(S)$. So $v$ separates two connected components in the induced subgraph by $\mathcal{U}(D)$, so $v$ is reachable under $D$. $s$ is 2-reachable under $\Delta_{D, D^{\prime}}$, thus $v$ is also 2-reachable under $D^{\prime}$ and $u$ is reachable.

$s$ was not reachable under $D$ hence its neighbors were not strongly reachable under $D$. Therefore $N[s] \subseteq \Delta \mathcal{T}_{D, D^{\prime}}$

Proof of Theorem 3.1. Indirectly assume that there is a graph $G$ such that $\pi_{\mathrm{opt}}(G)>\frac{15 n}{4(\delta+1)}$ and $\operatorname{diam}(G)>2$. This means that each solvable distribution has strengthening ratio below $\frac{4(\delta+1)}{15}$.

Let $D_{0}$ be a pebbling distribution which satisfies the properties of Lemma 3.5. Let $D$ be an expansion of $D_{0}$ such that the strengthening ratio of $D$ is at least $\frac{4(\delta+1)}{15}$ and subject to this requirement $|D|$ is maximal. According to our first assumption $D$ is not solvable. We will show that either $|D|$ is not maximal or $D$ is not an expansion of $D_{0}$. The first one is shown if we give a distribution $D^{\prime}$ such that $D<D^{\prime}$ and $\mathcal{E}_{D, D^{\prime}} \geq \frac{4(\delta+1)}{15}$. We will give $\Delta_{D, D^{\prime}}$ instead of $D^{\prime}$. Clearly $D$, and $\Delta_{D, D^{\prime}}$ together are determine $D^{\prime}$.

At each case we will assume that the conditions of the previous cases do not hold.

Case A: There exist $u, v \in \mathcal{U}(D)$ such that $d(u, v)=3$ and there is a vertex $w$ on a shortest path between $u$ and $v$ which is contained in $\mathcal{H}(D)$.

W.l.o.g. assume that $w$ is a neighbor of $v$. Then let $\Delta_{D, D^{\prime}}$ be the following:

$$
\Delta_{D, D^{\prime}}(x)= \begin{cases}4 & \text { if } x=u \\ 3 & \text { if } x=v \\ 0 & \text { otherwise }\end{cases}
$$

$v$ is 4-reachable under $D^{\prime}$, because $w$ is reachable under $D$ and it gets a pebble from $u$ under $\delta_{D, D^{\prime}}$, so $w$ is 2-reachable without the three pebbles of $v$. This means that each vertex of the closed neighborhood of $u$ and $v$ are strongly reachable.

$N[u]$ and $N[v]$ are disjoint vertex sets and they are subsets of $\Delta \mathcal{T}\left(D, D^{\prime}\right)$. Hence

$$
\mathcal{E}\left(D, D^{\prime}\right) \geq \frac{|N[u] \cup N[v]|}{\left|\Delta_{D, D^{\prime}}\right|} \geq \frac{2(\delta+1)}{7}>\frac{4}{15}(\delta+1),
$$

so $|D|$ was not maximal.

Case B: $\max _{u, v \in V(S)} d_{B}(u, v) \geq 4$

The conditions of case A are not satisfied, therefore there is a path in $S$ whose length is four in both $S$ and $B$ by Lemma 3.8 .

Apply Lemma 3.9 . If there are vertices $u$ and $v$ from $V(S)$ such that $d_{B}(u, v)=2$ and $\left|N_{B}[u] \cup N_{B}[v]\right| \geq \frac{28}{15}(\delta+1)$, then let $w$ be a common neighbor of $u$ and $v$ and choose $\Delta_{D, D^{\prime}}$ as follows:

$$
\Delta_{D, D^{\prime}}(x)= \begin{cases}2 & \text { if } x \in\{u, v\} \\ 3 & \text { if } x=w \\ 0 & \text { otherwise }\end{cases}
$$

Each of $u, v, w$ is 4-reachable, hence:

$$
\Delta t_{D, D^{\prime}} \geq|N[u] \cup N[v]| \geq \frac{28}{15}(\delta+1)
$$


$\left|\Delta_{D, D^{\prime}}\right|=7$, thus $\mathcal{E}\left(D, D^{\prime}\right) \geq \frac{4}{15}(\delta+1)$.

If there is no such an $u, v$ pair, then by Lemma 3.9 there is a path $a, b, c, d, e$ in $S$ such that $d_{B}(a, e)=$ $d_{S}(a, e)=4$, and $|N(b) \cap N(c)| \geq \frac{2}{15}(\delta+1)$. Consider $\Delta_{D, D^{\prime}}$ as follows:

$$
\Delta_{D, D^{\prime}}(x)= \begin{cases}4 & \text { if } x \in\{a, e\} \\ 0 & \text { otherwise }\end{cases}
$$

The vertices of set $N[a] \cup N[e] \cup(N[b] \cap N[d])$ are 2-reachable, thus they are also strongly reachable.

$$
\begin{gathered}
\Delta t \geq|N[a] \cup N[e] \cup(N[b] \cap N[d])|=|N[a]|+|N[e]|+|N[b] \cap N[d]| \geq \frac{32}{15}(\delta+1), \\
\mathcal{E}\left(D, D^{\prime}\right) \geq \frac{32(\delta+1)}{8 \cdot 15}=\frac{4(\delta+1)}{15} .
\end{gathered}
$$

Case C: $\max _{u, v \in V(S)} d_{B}(u, v)=3$.

If the conditions of Case $A$ do not hold, then we can use Lemma 3.7 because of Fact 3.6 . Let $(u, v)$ be the edge whose neighborhood size is at least $\left|\frac{4}{3}(\delta+1)\right|$. We will use this property only in the fourth subcase.

Consider the set $\mathcal{K}$, which is a set of vertex sets. $K$ is an element of $\mathcal{K}$ iff $K$ is a subset of $V(S)$ such that for all $k, j \in K, k \neq j$ implies that $d_{B}(k, j) \geq 3,|K| \geq 2$ and $K$ is maximal (we can not add an element to $K)$. $\max _{u, v \in V(S)} d_{B}(u, v)=3$ implies that $\mathcal{K}$ is not empty.

The objective in this case is to use Lemma 3.10 for the vertices of $K$. Because this means that the vertices of $\cup_{k \in K} N[k]$ are strongly reachable. Furthermore, $N\left[k_{1}\right]$ and $N\left[k_{2}\right]$ are disjoint if $k_{1}, k_{2} \in K$ and $k_{1} \neq k_{2}$. These imply that $\Delta t \geq \cup_{k \in K}|N(k)| \geq|K|(\delta+1)$. To use this Lemma we need to give a proper $\Delta_{D, D^{\prime}}$ distribution and check that each vertex of $S$ is reachable and each vertex of $K$ is 2-reachable under it.

There are four subcases here:

Subcase 1: $\forall s \in V(S) d_{B}(v, s) \leq 2$.

Let $K$ be an arbitrary element of $\mathcal{K}$. Note that $v \notin K$.

$$
\Delta_{D, D^{\prime}}(x)= \begin{cases}4 & \text { if } x=v \\ 1 & \text { if } x \in K \\ 0 & \text { otherwise }\end{cases}
$$

Each vertex of $S$ is reachable with the pebbles placed at $v$ and the vertices of $K$ are 2-reachable.

$$
\mathcal{E}\left(D, D^{\prime}\right) \geq \frac{|K|(\delta+1)}{4+|K|} \geq \frac{1}{3}(\delta+1) .
$$

Subcase 2: $\forall s \in V(S) \min \left(d_{B}(u, s), d_{B}(v, s)\right) \leq 2$, but $\exists w \in V(S) d_{B}(v, w)=3$.

Choose $K$ such that $v \in K$. Such a $K$ is exists.

$$
\Delta_{D, D^{\prime}}(x)= \begin{cases}3 & \text { if } x \in\{u, v\}, \\ 1 & \text { if } x \in K \backslash\{v\}, \\ 0 & \text { otherwise }\end{cases}
$$

$u$ and $v$ are 4-reachable, hence all vertices of $S$ are reachable. Furthermore, each vertex of $K$ is 2reachable.

$$
\mathcal{E}\left(D, D^{\prime}\right) \geq \frac{|K|(\delta+1)}{6+|K|-1} \geq \frac{2}{7}(\delta+1) .
$$

Subcase 3: $\exists s \in V(S) d_{B}(s, u)=d_{B}(s, v)=3$ and $\{s, v\} \notin \mathcal{K}$. 
$\{s, v\}$ is a subset of some elements of $\mathcal{K}$. Choose $K$ as one of these, $|K| \geq 3$.

$$
\Delta_{D, D^{\prime}}(x)= \begin{cases}8 & \text { if } x=v, \\ 1 & \text { if } x \in K \backslash\{v\}, \\ 0 & \text { otherwise }\end{cases}
$$

Each vertex of $S$ is reachable with the pebbles placed at $v$ and the vertices of $K$ are 2-reachable.

$$
\mathcal{E}\left(D, D^{\prime}\right) \geq \frac{|K|(\delta+1)}{8+|K|-1} \geq \frac{3}{10}(\delta+1)
$$

Subcase 4: $\exists s \in V(S) d_{B}(s, u)=d_{B}(s, v)=3$ and $\{s, v\} \in \mathcal{K}$.

$K=\{s, v\}$

$$
\Delta_{D, D^{\prime}}(x)= \begin{cases}4 & \text { if } x \in K, \\ 0 & \text { otherwise }\end{cases}
$$

$K=\{s, v\}$ means that each vertex of $S$ is in $N^{2}[s] \cup N^{2}[v]$, thus each vertex of $S$ is reachable. $N[s] \cap$ $(N[u] \cup N[v])=\emptyset$ hence:

$$
\begin{gathered}
\Delta t_{D, D^{\prime}} \geq|N[s] \cup N[v] \cup N[u]|=|N[s]|+|N[v] \cup N[u]| \geq \frac{7}{3}(\delta+1), \\
\mathcal{E}\left(D, D^{\prime}\right) \geq \frac{7}{24}(\delta+1) .
\end{gathered}
$$

Case D: $\max _{u, v \in V(S)} d_{B}(u, v) \leq 2$.

In this case if we put 4 pebbles to an arbitrary vertex $s$ of $S$, then all vertices of $S$ and $N[s]$ becomes strongly reachable.

Subcase 1: $|V(S)| \geq \frac{16}{15}(\delta+1)$.

Let $v$ be a vertex of $S$.

$$
\begin{gathered}
\Delta_{D, D^{\prime}}(x)= \begin{cases}4 & \text { if } x=v, \\
0 & \text { otherwise. }\end{cases} \\
\mathcal{E}\left(D, D^{\prime}\right) \geq \frac{16(\delta+1)}{15 \cdot 4}=\frac{4}{15}(\delta+1)
\end{gathered}
$$

Subcase 2a: $\exists u, v \in V(s)$ such that $|N[u] \cup N[v]| \geq \frac{16}{15}(\delta+1)$ and $u$ and $v$ are neighbors.

$$
\Delta_{D, D^{\prime}}(x)= \begin{cases}4 & \text { if } x=v \\ 0 & \text { otherwise }\end{cases}
$$

Each vertex of $S$ is reachable and $u$ and $v$ are 2-reachable under $\Delta_{D, D^{\prime}}$. Using Lemma 3.10 we get that the neighborhoods of $u$ and $v$ are both strongly reachable.

$$
\mathcal{E}\left(D, D^{\prime}\right) \geq \frac{|N[u] \cup N[v]|}{4} \geq \frac{16(\delta+1)}{15 \cdot 4}=\frac{4}{15}(\delta+1) .
$$

Subcase 2b: $\exists u, v \in V(s)$ such that $|N[u] \cup N[v]| \geq \frac{16}{15}$ and $u$ and $v$ share a common neighbor $w \in V(s)$.

$$
\Delta_{D, D^{\prime}}(x)= \begin{cases}4 & \text { if } x=w \\ 0 & \text { otherwise }\end{cases}
$$

We can say the same like in the previous case.

Subcase 3a: $|V(S)| \leq \frac{14}{15}(\delta+1)$ and $\forall u, v \in V(S) \exists h \in \mathcal{H}(D) h \in N(u) \cap N(v)$. 
Choose $v$ as an arbitrary vertex of $S$.

$$
\Delta_{D, D^{\prime}}(x)= \begin{cases}2 & \text { if } x=v, \\ 0 & \text { otherwise }\end{cases}
$$

If $s$ is a vertex of $S$ other than $v$, then there is $h \in \mathcal{H}(D)$ which is a neighbor of both of them. $h$ is reachable under $D$, under $D^{\prime}$ we have two additional pebbles so we can move a pebble to $s$ from $v$ through $h$. Thus each vertex of $S$ is reachable under $D^{\prime}$, so we can apply Lemma 3.10 for vertex $v$.

$$
\mathcal{E}\left(D, D^{\prime}\right) \geq \frac{|N[v]|}{2} \geq \frac{\delta+1}{2} .
$$

Subcase 3b: $|V(S)| \leq \frac{14}{15}(\delta+1)$ and $\exists u, v \in V(S) \nexists h \in \mathcal{H}(D) h \in N(u) \cap N(v)$.

The diameter of $S$ (with respect to the distance defined in $B$ ) guarantees that either $u$ and $v$ are neighbors or they share a common neighbor $w \in V(B)$. Furthermore, in this subcase $w \in V(S)$. $u$ has at least $\delta-\left(\frac{14}{15}(\delta+1)-1\right)=\frac{1}{15}(\delta+1)$ neighbors in $\mathcal{H}(D)$, but none of them is a neighbor of $v$. Hence $|N[u] \cup N[v]| \geq \frac{16}{15}(\delta+1)$. This is subcase $2 \mathrm{a}$ or $2 \mathrm{~b}$.

Subcase 4: $\exists v \in V(S)$, such that $\forall s \in V(S) d_{B}(v, s)=1$.

$$
\Delta_{D, D^{\prime}}(x)= \begin{cases}2 & \text { if } x=v, \\ 0 & \text { otherwise. }\end{cases}
$$

Each vertex of $S$ is reachable under $D^{\prime}$ so we apply Lemma 3.10 again and get that $\mathcal{E}\left(D, D^{\prime}\right) \geq \frac{\delta+1}{2}$.

We have handled all cases when $|V(S)| \geq \frac{14}{15}(\delta+1)$ or $|V(S)| \leq \frac{16}{15}(\delta+1)$. So in the next sections we assume that $\frac{14}{15}(\delta+1)<|V(S)|<\frac{16}{15}(\delta+1)$. Before we continue, we need one more definition. Let $\mathcal{S}$ be the set of connected components of the graph which is induced by $\mathcal{U}(D)$. Then we say that $S \in \mathcal{S}$ is isolated in $\mathcal{S}$ if for any other $S^{\prime}$ in $\mathcal{S} d_{G}\left(S, S^{\prime}\right) \geq 3$.

Subcase 5: $\exists S \in \mathcal{S}$ such that $S$ is not isolated.

Exists $S^{\prime}$ and $u \in S, v \in S$ such that $d(u, v)=2$.

$$
\Delta_{D, D^{\prime}}(x)= \begin{cases}4 & \text { if } x=u \\ 3 & \text { if } x=v \\ 0 & \text { otherwise }\end{cases}
$$

$u$ and $v$ are both 4-reachable, hence all vertices of $S$ and $S^{\prime}$ are reachable, furthermore they are strongly reachable.

$$
\mathcal{E}\left(D, D^{\prime}\right) \geq \frac{\left|V(S) \cup V\left(S^{\prime}\right)\right|}{7} \geq \frac{2 \cdot \frac{14}{15}(\delta+1)}{7}=\frac{4(\delta+1)}{15}
$$

Subcase 6: $S$ is isolated in $\mathcal{S}$ and $|N[S]| \geq \frac{16}{15}(\delta+1)$.

Let $s$ be an arbitrary vertex of $S$. Then:

$$
\Delta_{D, D^{\prime}}(x)= \begin{cases}4 & \text { if } x=s \\ 0 & \text { otherwise }\end{cases}
$$

Each vertex of $S$ becomes strongly reachable. We show that the same is true for any vertex of $N(S)$. Consider $h \in N(S)$. $h$ is not strongly reachable under $D$, but all of its non reachable neighbors under $D$ are contained in $S$, because $S$ is isolated. Thus under $D^{\prime} h$ is strongly reachable which gives the following result:

$$
\mathcal{E}\left(D, D^{\prime}\right) \geq \frac{|N[V(S)]|}{4} \geq \frac{\frac{16}{15}(\delta+1)}{4}=\frac{4(\delta+1)}{15} .
$$


Subcase 7: $S$ is isolated in $\mathcal{S}$ and $\exists h \in \mathcal{H}(D)$ such that for each $s \in V(S) d_{G}(h, s) \leq 2$.

$$
\Delta_{D, D^{\prime}}(x)= \begin{cases}3 & \text { if } x=h, \\ 0 & \text { otherwise }\end{cases}
$$

Each vertex of $S$ becomes strongly reachable thus:

$$
\mathcal{E}\left(D, D^{\prime}\right) \geq \frac{|V(S)|}{3}>\frac{\frac{14}{15}(\delta+1)}{3} \geq \frac{4(\delta+1)}{15} .
$$

Subcase 8: None of the previous cases hold.

In this case we will get a contradiction with $D_{0} \leq D$. We summarize what we know about $D$ :

- $\forall S \in \mathcal{S} \max _{u, v \in V(S)}\left(d_{B}(u, v)\right)=2$,

- $\forall S \in \mathcal{S} \frac{14}{15}(\delta+1)<|V(S)|<\frac{16}{15}(\delta+1)$,

- $\forall S$ is isolated in $\mathcal{S}$,

- $\forall S \in \mathcal{S}|N[S]|<\frac{16}{15}(\delta+1)$,

- $\nexists h \in \mathcal{H}(D)$ such that the distance between $h$ and any vertex of $S$ is at most two, where $S \in \mathcal{S}$.

First of all, the diameter of $G$ is at least 3, hence some pebbles have been placed, so $\mathcal{H}(D)$ is nonempty. Fix a component $S \in \mathcal{S}$. $\mathcal{H}(D) \cap N(S)$ is also nonempty, because $G$ is connected. Consider an $h \in$ $\mathcal{H}(D) \cap N(S)$. The last property guarantees that there is a vertex $v$ in $V(S)$ such that $d(v, h)=3$. A neighbour of $h$ is in $V(S)$. Denote this vertex with $u$. The first property and $d(v, h)=3$ together imply that $u \in N^{2}(v) \cap S$.

$h$ is in $N^{3}(v)$, hence $N[h] \cap N[v]=\emptyset . N[v] \subseteq N[S]$ :

$$
|N[h] \cap N[S]| \leq|N[S] \backslash N[v]|=|N[S]|-|N[v]|<\frac{16}{15}(\delta+1)-(\delta+1)=\frac{1}{15}(\delta+1) .
$$

So we have: $|N[h] \backslash N[S]| \geq \frac{14}{15}(\delta+1)$. $u$ is contained in $S$, hence all of its neighbors are contained in $N[S]$, thus:

$$
|N[u, h]| \geq|N[u]|+|N[h] \backslash N[S]| \geq \frac{29}{15}(\delta+1) .
$$

$u$ is in $S$, so it is not reachable under $D$, but $D$ is an expansion of $D_{0}$ where $u$ has to be reachable because $|N[u, h]| \geq \frac{29}{15}(\delta+1)$ and $(u, h) \in E(G)$. This is a contradiction.

We have seen that in each case we have a contradiction, so our assumption was false, hence the theorem is true.

Muntz et al. [10] characterize diameter three graph graphs whose optimal pebbling number is eight. Their characterization can be reformulated in the following weird statement.

Claim 3.11 Let $G$ be a diameter 3 graph. $\pi_{\mathrm{opt}}(G)=8$ if and only if there are no vertices $x, u, v$ and $w$ such that $N^{2}[x] \cup N[u] \cup N[v] \cup N[w]=V(G)$.

Theorem 3.1 can be used to establish a connection between this unusual domination property and the minimum degree of the graph. Note that this is just a minor improvement of the trivial $\frac{1}{2} n-1$ upper bound.

Corollary 3.12 Let $G$ be a diameter 3 graph on $n$ vertices. If there are no vertices $x, u, v$ and $w$ such that $N^{2}[x] \cup N[u] \cup N[v] \cup N[w]=V(G)$, then the minimum degree of $G$ is at most $\frac{15}{32} n-1$. 


\section{The diameter two case}

Theorem 4.1 For any $\epsilon>0$ there is a graph $G$ with diameter two, such that $\pi_{\text {opt }}(G)>\frac{(4-\epsilon) n}{\delta+1}$.

The optimal pebbling number of a diameter two graph which does not have a dominating edge is 4 . We show, with the help of random graphs, that such a graph exists with high minimum degree.

Erdös' probabilistic method is a commonly used technique to show the existence of an object without its explicit construction. Random graphs are investigated by many great mathematicians. In case the reader is not familiar with this topic we recommend the book of Bollobás [2] and a recent book of Frieze and Karoński [8].

Let $G(n, p)$ be an Erdős-Rényi random graph on $n$ vertices where two vertices are adjacent with probability $p$. Assume in the rest of the paper that $0<p<1$ and $q=1-p$.

Claim 4.2 If $p$ is fixed, then for sufficiently large $n G(n, p)$ does not contain a dominating edge with probabilty higher than 0.9 .

Proof: Let $Y$ be the number of dominating edges in $G(n, p)$.

$$
\begin{aligned}
\mathbb{E} Y & =\mathbb{E} \sum_{e \in E\left(K_{n}\right)} \mathbb{1}\{e \text { is a dominating edge in } G(n, p)\}=\sum_{e \in E\left(K_{n}\right)} \mathbb{P}(e \text { is a dominating edge in } G(n, p))= \\
& =\sum_{\substack{u, v \in V\left(K_{n}\right) \\
u<v}} \mathbb{P}(\{u, v\} \in E(G(n, p))) \mathbb{P}(u, v \text { dominates } G(n, p))=\left(\begin{array}{l}
n \\
2
\end{array}\right) p\left(1-q^{2}\right)^{n-2} \underset{n \rightarrow \infty}{\longrightarrow} 0
\end{aligned}
$$

Using Markov's inequality:

$$
\mathbb{P}(\text { a dominating edge exists })=\mathbb{P}(Y \geq 1) \leq \mathbb{E} Y \underset{n \rightarrow \infty}{\longrightarrow} 0
$$

Claim 4.3 If $p$ is fixed, then for sufficiently large $n$ the diameter of $G(n, p)$ is two with probabilty higher than 0.9 .

Proof: Let $Z$ be the number of quadruples from $V(G(n, p))$ such that the quadruple induces a $P_{4}$ and the two endpoints of this $P_{4}$ does not share a neighbour. Note that a graph whose diameter is at least three contains such a quadruple.

$$
\begin{aligned}
\mathbb{E} Z & =\mathbb{E} \sum_{\substack{u, v, w, x \in V\left(K_{n}\right) \\
u<x}} \mathbb{1}\left\{u, v, w, x \text { induces a } P_{4} \text { in this order in } G(n, p) \text { and } \nexists y \in N(u) \cap N(x)\right\}= \\
& =\sum_{u, v, w, x \in V\left(K_{n}\right)} \mathbb{P}\left(u, v, w, x \text { induces a } P_{4} \text { in this order in } G(n, p) \text { and } \nexists y \in N(u) \cap N(x)\right)= \\
& =\left(\begin{array}{c}
n \\
4
\end{array}\right) p^{3} q^{3}\left(1-p^{2}\right)^{n-4} \underset{n \rightarrow \infty}{\longrightarrow} 0
\end{aligned}
$$

Using Markov's inequality again:

$$
\mathbb{P}(\operatorname{diam}(G(n, p)) \geq 3)=\mathbb{P}(Z \geq 1) \leq \mathbb{E} Z \underset{n \rightarrow \infty}{\longrightarrow} 0
$$

The only graph with diameter one on $n$ vertices is $K_{n}$, hence

$$
\lim _{n \rightarrow \infty} \mathbb{P}(\operatorname{diam}(G(n, p))=2)=1-\lim _{n \rightarrow \infty} \mathbb{P}(\operatorname{diam}(G(n, p)) \geq 3)=1
$$


It is known that the maximum and minimum degrees of $G(n, p)$ are concentrated around $n p$ when $n$ tends to infinity. More precisely, using a multiplicative form of Chernoff's bound [1, Proposition 2.4], the following can be proved:

$$
\mathbb{P}(\exists v \operatorname{deg}(v) \leq(1-\tilde{\epsilon})(n-1) p) \leq n \exp \left(\frac{-(n-1) p \tilde{\epsilon}^{2}}{2}\right) \underset{n \rightarrow \infty}{\longrightarrow} 0
$$

So for large $n$ the minimum degree is at least $(1-\tilde{\epsilon})(n-1) p$ with high probabilty.

Proof of Theorem 4.1. Choose $\tilde{\epsilon}$ and $p$ as $\frac{\epsilon}{8}$ and $\left(1-\frac{\epsilon}{4}\right) /\left(1-\frac{\epsilon}{8}\right)$, respectively. Let $n$ be large enough such that we can apply the three previous results. So we have a graph $G$ on $n$ vertices such that it does not have a domination edge, its diameter is two and its minimum degree is at least $(1-\tilde{\epsilon})(n-1) p$. Then:

$$
\frac{(4-\epsilon) n}{\delta+1} \leq \frac{(4-\epsilon) n}{(1-\tilde{\epsilon})(n-1) p+1}<\frac{(4-\epsilon) n}{\left(1-\frac{\epsilon}{8}\right) p n}=4=\pi_{\mathrm{opt}}(G)
$$

Claim 4.4 There is no connected graph $G$ such that $\pi_{\text {opt }}(G)=\frac{4 n}{\delta+1}$.

Proof: Theorem 3.1 shows that the optimal pebbling number of graphs whose diameter is at least three is smaller. So we have to check only diameter two and complete graphs whose optimal pebbling number is at most $4 . \frac{4 n}{\delta+1} \geq 4$ and equality holds only for the complete graph, but $\pi_{o p t}\left(K_{n}\right)=2$.

Corollary 4.5 For any connected graph $G \pi_{\text {opt }}(G)<\frac{4 n}{\delta+1}$ and this bound is sharp.

\section{Acknowledgment}

The research of Gyula Y. Katona is partially supported by National Research, Development and Innovation Office NKFIH, grant K116769. The research of Gyula Y. Katona and László F. Papp is partially supported by National Research, Development and Innovation Office NKFIH, grant K108947.

\section{References}

[1] D. Angluin, L. G. Valiant Fast probabilistic algorithms for hamiltonian circuits and matchings Journal of Computer and System Sciences 182 (1979) pp. 155-193

[2] B. Bollobás Random Graphs Cambridge University Press (2001)

[3] F. Chung, Pebbling in hypercubes SIAM J. Discrete Math. 2 (1989) pp. 467-472

[4] D.P. Bunde, E. W. Chambers, D. Cranston, K. Milans, D. B. West, Pebbling and optimal pebbling in graphs J. Graph Theory 57 no. 3. (2008) pp. 215-238.

[5] H. Fu, C. Shiue, The optimal pebbling number of the caterpillar, Taiwanese Journal of Mathematics, 13 no. 2A (2009) pp. 419-429

[6] H. Fu, C. Shiue, The optimal pebbling number of the complete m-ary tree, Discrete Mathematics, 222 1-3 (2000) pp. 89-100 
[7] T. Friedman, C.Wyels. Optimal pebbling of paths and cycles arXiv:math/0506076 [math.CO]

[8] A. Frieze, M. Karoński Introduction to Random Graphs Cambridge University Press (2016)

[9] K. Milans, B. Clark, The complexity of graph pebbling SIAM J. Discrete Math. 20 no. 3 (2006) pp. 769-798.

[10] J. Muntz, S. Narayan, N. Streib, K. V. Ochten Optimal pebbling of graphs Discrete Mathematics, 307 (2007) pp. 2315-2321

[11] L. Pachter, H.S. Snevily, B. Voxman On pebbling graphs Congressus Numerantium, 107 (1995) pp. 65-80.

[12] C. Wyels, T. Friedman, Optimal pebbling of paths and cycles arXiv:math/0506076 